\title{
Subsonic propellers in a strong wind as anomalous X-ray pulsars
}

\author{
N. R. Ikhsanov ${ }^{1,2,3}$ and C.-S. Choi ${ }^{1}$ \\ 1 Korea Astronomy Observatory, 61-1 Hwaam, Yusong, Taejon 305-348, Korea \\ e-mail: ikhsanov@kao.re.kr \\ 2 Central Astronomical Observatory of the Russian Academy of Sciences, Pulkovo 65-1, 196140 Saint-Petersburg, Russia \\ ${ }^{3}$ Isaac Newton Institute of Chile, St. Petersburg Branch, Russia
}

Received 21 April 2004 / Accepted 11 May 2004

\begin{abstract}
The appearance of subsonic propellers situated in a strong wind is discussed. We show that it is similar to the appearance of anomalous X-ray pulsars (AXPs) provided the mass and the magnetic moment of neutron stars are $M \simeq 1.4 M_{\odot}$, and $\mu \simeq 2 \times 10^{30} \mathrm{G} \mathrm{cm}^{3}$, respectively, and the strength of the wind is $\dot{M}_{\mathrm{c}} \simeq 3 \times 10^{17} \mathrm{~g} \mathrm{~s}^{-1}$. Under these conditions, the spin periods of subsonic propellers are limited within the range of $5-15 \mathrm{~s}$, and the expected spin-down rates are close to $\dot{P} \simeq$ $7 \times 10^{-11} \mathrm{~s} \mathrm{~s}^{-1}$. The mass accretion rate onto the stellar surface is limited to the rate of plasma penetration into its magnetosphere at the boundary. As this process is governed by the reconnection of the field lines, the accretion rate onto the stellar surface constitutes $1-2 \%$ of $\dot{M}_{\mathrm{c}}$. In this case the X-ray luminosity of the objects under consideration can be evaluated as $L_{\mathrm{X}} \sim 4 \times$ $10^{35} \mathrm{erg} \mathrm{s}^{-1}$. The model predicts the existence of at least two spatially separated sources of the X-ray emission: hot spots at the stellar surface, and the hot atmosphere surrounding the magnetosphere of the star. The ages of the subsonic propellers under the conditions of interest are limited to $\lesssim 10^{5} \mathrm{yr}$.
\end{abstract}

Key words. accretion, accretion disks - stars: neutron - stars: winds, outflows - X-rays: stars

\section{Introduction}

Anomalous X-ray pulsars (AXPs) are currently recognized as a separate class of single magnetized neutron stars, with common characteristics different from those of the normal pulsars. The theoretical interpretation of these objects is still a point of discussion. A successful theoretical model of AXPs should be able to explain the following basic properties of these objects (see Mereghetti et al. 2002 for a comprehensive review):

(1) the clustering of spin periods in the range 5-12 s;

(2) the spin-down rates of $10^{-10}-10^{-12} \mathrm{~s} \mathrm{~s}^{-1}$;

(3) the soft X-ray spectra, which are clearly different from those of the accretion-powered pulsars;

(4) the X-ray luminosity at a level of $\sim 10^{35}-10^{36} \mathrm{erg} \mathrm{s}^{-1}$;

(5) relatively young ages of these objects, derived from the association of at least two of AXPs with supernova shell remnants (SNRs).

A key question in the modeling of AXPs is the state of the neutron star. Actually, there are only 4 possible answers: ejector, supersonic propeller, subsonic propeller, and accretor (see e.g. Ikhsanov 2001a). Three of these possibilities have already been discussed in the literature. The state of ejector (=spinpowered pulsar) was analyzed within the magnetar model. The observed spin-down rates, the soft spectra and the young ages of AXPs can be interpreted within this model provided the strength of the dipole magnetic field of the star is $B \gtrsim 10^{15} \mathrm{G}$ (see e.g. Thompson \& Duncan 1996 and references therein). At the same time, the clustering of the periods can hardly be explained in terms of magnetars unless some deviations from the canonical scheme of the evolution of neutron stars are invoked in the model (for discussion see Psaltis \& Miller 2002; Colpi et al. 2000).

The excess of the observed luminosity over the spin-down power allows us to reject the possibility that AXPs are supersonic propellers, i.e. spinning-down neutron stars for which the condition $R_{\text {cor }} \lesssim R_{\mathrm{m}}<\min \left\{R_{\alpha}, R_{\mathrm{lc}}\right\}$ is satisfied. Here $R_{\text {cor }}=\left(G M_{\mathrm{ns}} / \omega^{2}\right)^{1 / 3}, R_{\alpha}=2 G M_{\mathrm{ns}} / V_{\text {rel }}^{2}$ and $R_{\mathrm{m}}$ are the corotation, accretion, and magnetospheric radii of the neutron star, respectively. $R_{\mathrm{lc}}=c / \omega$ is the radius of the light cylinder. $\omega=2 \pi / P_{\mathrm{s}}$ and $M_{\mathrm{ns}}$ denote the angular velocity and the mass of the star, and $V_{\text {rel }}$ is the relative velocity between the star and the surrounding medium. $G$ and $c$ are the gravitational constant and the speed of light. On this basis, the corresponding state is presently discussed (e.g. Marsden et al. 2001) only in the context of a previous spin-down evolution of AXPs to their present state.

The modeling of AXPs as neutron stars accreting material from a disk has been reported by many authors (see e.g. Chatterjee et al. 2000; Mereghetti et al. 2002, for a detailed description). Within this approach, the observed luminosity is interpreted in terms of the accretion power. Therefore, the "power deficit" problem mentioned above turns out to be naturally avoided. However, to meet other criteria, such as the relatively high spin-down rates, the soft X-ray spectrum and the 
clustering of periods in a relatively narrow range, a rather specific scheme of neutron star evolution has to be invoked (Eksi \& Alpar 2003).

In the light of this situation, a consideration of the last possibility, namely, that the state of neutron stars in AXPs is subsonic propeller appears quite reasonable. The corresponding analysis is the subject of this paper. As shown below, the modeling of AXPs within the subsonic propeller approach appears effective in several important aspects. In particular, it provides us with an explanation of the observed clustering of periods (Sect. 2) and spin-down rates (Sect. 3). It also predicts the existence of two spatially separated sources of X-ray emission (Sect. 4) with the total luminosity of order of the observed luminosity of AXPs. The ages of supersonic propellers are evaluated in Sect. 5. The proposed scenario is discussed in Sect. 6 and the basic conclusions are summarized in Sect. 7.

\section{Period clustering}

As recently shown by Ikhsanov (2001b), the spin period of a neutron star in the state of subsonic propeller satisfies the following condition: $P_{\mathrm{cd}} \lesssim P_{\mathrm{s}} \lesssim P_{\mathrm{br}}$, where

$P_{\mathrm{cd}} \simeq 3.2 \mu_{30}^{6 / 7} m^{-5 / 7} \dot{M}_{17}^{-3 / 7} \mathrm{~s}$,

is the period at which the star switches its state from supersonic to subsonic propeller, and

$P_{\mathrm{br}} \simeq 16 \mu_{30}^{16 / 21} \dot{M}_{17}^{-5 / 7} m^{-4 / 21} \mathrm{~s}$,

is the period at which the state of the star switches to accretor. Here $m$ and $\mu_{30}$ are the mass and the dipole magnetic moment of the star expressed in units of $M_{\odot}$, and $10^{30} \mathrm{G} \mathrm{cm}^{3}$, respectively. $\dot{M}_{17}$ is the strength of the wind, $M_{\mathrm{c}}$, expressed in units of $10^{17} \mathrm{~g} \mathrm{~s}^{-1}$. The meaning of this parameter is the mass of the surrounding material interacting with the neutron star, moving through the medium with the velocity $V_{\text {rel }}$, in a time unit: $\dot{M}_{\mathrm{c}}=\pi R_{\alpha}^{2} \rho_{\infty} V_{\text {rel }}$. Here $\rho_{\infty}$ is the density of material surrounding the star at the distance $R_{\alpha}$.

Combining Eqs. (1) and (2) we find that the clustering of periods of AXPs can be explained in terms of the subsonic propeller approach provided these objects are neutron stars of mass $M \sim 1.4 M_{\odot}$ and magnetic moment $\mu \sim 2 \times 10^{30} \mathrm{G} \mathrm{cm}^{3}$, situated in a wind of strength $\dot{M}_{\mathrm{c}} \sim 3 \times 10^{17} \mathrm{~g} \mathrm{~s}^{-1}$.

\section{Spin-down rate}

As shown by Davies \& Pringle (1981), the spin-down power of stars in the state of subsonic propeller can be evaluated as

$L_{\mathrm{ssp}}=8 \times 10^{33} \mu_{30}^{2} \mathrm{~m}^{-1} P_{5}^{-3} \operatorname{egr~s}^{-1}$,

where $P_{5}=P_{\mathrm{s}} / 5 \mathrm{~s}$. This means that subsonic propellers are expected to spin down at a rate

$\dot{P}=\frac{P_{\mathrm{s}}^{3} L_{\mathrm{ssp}}}{4 \pi^{2} I} \sim 2.5 \times 10^{-11} \mu_{30}^{2} m^{-1} I_{45}^{-1} \mathrm{~s} \mathrm{~s}^{-1}$,

where $I_{45}$ is the moment of inertia of the neutron star expressed in units of $10^{45} \mathrm{~g} \mathrm{~cm}^{2}$.

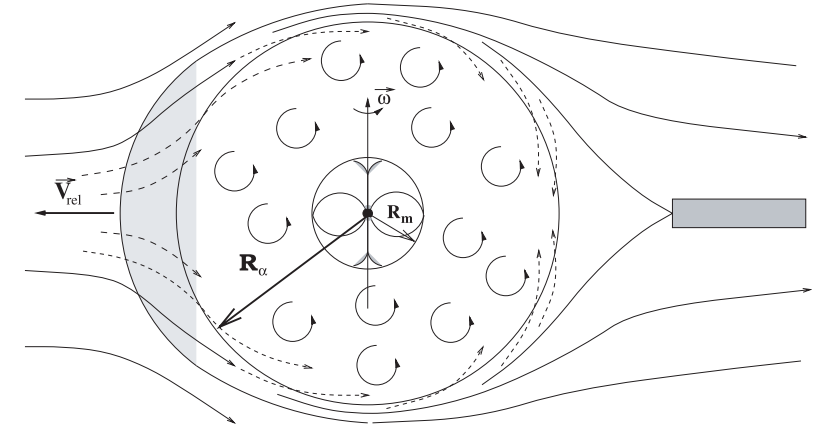

Fig. 1. Schematic description of the subsonic propeller. A magnetized neutron star rotates with angular velocity $\omega$. The magnetosphere of the star is surrounded by a hot turbulent envelope. As the star moves through the medium with relative velocity $\boldsymbol{V}_{\text {rel }}$, the gas overflows the outer edge of the envelope compressing the envelope plasma. The overflow rate is equal to the wind strength, $\dot{M}_{\mathrm{c}}$. The rate of plasma motion through the envelope is equal to the rate of plasma penetration into the magnetosphere of the star at the inner boundary of the envelope, $\dot{M}_{\text {rec }}$. The material penetrating into the magnetic field of the star flows along the field lines and reaches the stellar surface at the magnetic poles. The hatched regions represent shocks in front of and behind the subsonic propeller moving through the dense environment.

Putting the above derived parameters $\left(M \sim 1.4 M_{\odot}\right.$ and $\mu \sim$ $2 \times 10^{30} \mathrm{G} \mathrm{cm}^{3}$ ) into Eq. (4), one finds that the spin-down rate of AXPs within the subsonic propeller approach is expected to be of order $7 \times 10^{-11} \mathrm{~s} \mathrm{~s}^{-1}$. This is in a good agreement with the typically observed values.

\section{Accretion power}

According to the picture presented by Davies \& Pringle (1981), the magnetosphere of a neutron star in the state of subsonic propeller is surrounded by an adiabatic $\left(p \propto R^{-5 / 2}\right)$ spherically symmetrical plasma envelope. The energy input to the envelope due to the propeller action by the star dominates the energy losses from the envelope as long as $P_{\mathrm{s}}<P_{\mathrm{br}}$. Therefore, the temperature of the envelope plasma remains of order of the free-fall temperature, $T_{\mathrm{pl}} \simeq T_{\mathrm{ff}}=G M_{\mathrm{ns}} m_{\mathrm{p}} / k R$ and, correspondingly, the sound speed is of order of the free-fall velocity, $V_{\mathrm{s}} \simeq V_{\mathrm{ff}}=\sqrt{2 G M_{\mathrm{ns}} / R}$ (here $m_{\mathrm{p}}$, and $k$ are the proton mass and the Boltzmann constant). Under these conditions the height of the homogeneous atmosphere throughout the envelope is comparable to the radius, $R$, and the envelope is extended from the magnetospheric boundary up to the accretion radius of the star.

Within this picture (see Fig. 1) the envelope is quasi-static: the characteristic time of the drift of material through the envelope in the radial direction is much larger than the characteristic time of the turbulent motions. The rate of the drift is significantly smaller than $\dot{M}_{\mathrm{c}}$, i.e. the rate by which the circumstellar material overflows the outer edge of the envelope compressing the envelope plasma (for discussion see Ikhsanov 2003, and references therein).

Under the condition $P_{\mathrm{s}}>P_{\mathrm{cd}}$, the centrifugal barrier is not effective: the plasma, penetrating from the envelope into the magnetic field of the star, is able to flow along the magnetic field lines and to accrete onto the stellar surface. Therefore, an accretion process onto the stellar surface can be expected. 
However, the rate of this process is limited to the rate of plasma penetration into the magnetic field of the star.

As shown by Arons \& Lea (1976a) and Elsner \& Lamb (1976), the interchange (e.g. Rayleigh-Taylor) instabilities of the magnetospheric boundary under the condition $T_{\mathrm{pl}} \sim T_{\mathrm{ff}}$ are suppressed (we would like to note that the observed luminosities of AXPs are a factor of 5-10 lower than the critical luminosity for which Compton cooling is effective). In this situation, the process of plasma penetration into the magnetosphere is governed by the Bohm diffusion and/or the reconnection of the field lines at the magnetospheric boundary. An evaluation of the corresponding rates of penetration has recently been reported by Ikhsanov (2001a, see Eqs. (22) and (24)). Using these results, one finds that the rate of plasma penetration into the magnetosphere due to the reconnection of the field lines is high enough for the X-ray luminosity of subsonic propellers to be of the order of that observed from AXPs (see Eq. (24) in Ikhsanov 2001a)

$L_{\mathrm{acc}}=\dot{M}_{\mathrm{rec}} \frac{G M_{\mathrm{ns}}}{R_{\mathrm{ns}}} \sim 4 \times 10^{35} \mathrm{erg} \mathrm{s}^{-1}$

$\times\left[\frac{M_{\mathrm{ns}}}{1.4 M_{\odot}}\right]\left[\frac{R_{\mathrm{ns}}}{10^{6} \mathrm{~cm}}\right]^{-1}\left[\frac{\alpha_{\mathrm{r}}}{0.1}\right]\left[\frac{\lambda_{\mathrm{m}}}{0.1 R_{\mathrm{m}}}\right]\left(\frac{\dot{M}_{\mathrm{c}}}{3 \times 10^{17} \mathrm{~g} \mathrm{~s}^{-1}}\right)$.

Here $\dot{M}_{\text {rec }}$ is the rate of plasma penetration from the envelope into the magnetosphere due to the reconnection process, and $R_{\mathrm{ns}}$ is the radius of the neutron star. $\alpha_{\mathrm{r}}$ is the efficiency of the reconnection process, which is normalized according to the results of modeling of the reconnection processes in solar flares and in the Earth's magnetopause (see Priest \& Forbes 2000 and references therein). $\lambda_{\mathrm{m}}$ is the average scale of plasma inhomogeneities at the base of the envelope, which is normalized following Arons \& Lea (1976b), and Wang \& Robertson (1985).

This indicates that the radiation of subsonic propellers situated in a strong wind is powered mainly by the reconnectiondriven accretion of material onto the stellar surface. At the same time, from 5 to 10 percent of the radiation emitted by these objects are expected to be contributed by the hot envelope surrounding the magnetosphere (see Eq. (3)). Therefore, within the considered approach the existence of at least two spatially separated sources of the X-ray emission can be expected.

A detailed analysis of the radiation spectrum of subsonic propellers is beyond the scope of this paper and will be presented in a forthcoming paper.

\section{Age}

The age of subsonic propellers can be expressed as the superposition of pulsar-like and supersonic propeller spin-down time scales.

\subsection{Pulsar-like spin-down time scale}

The period at which the neutron star switches its state from ejector to supersonic propeller is (see Eq. (6) in Ikhsanov 2001a)

$P_{\mathrm{md}}=0.2 \mu_{30.3}^{1 / 2} \dot{M}_{17.5}^{-1 / 4} V_{7}^{-1 / 4} \mathrm{~s}$.
The spin-down rate of the star in the ejector state is

$\dot{P}=\frac{8 \pi^{2} \mu^{2}}{3 c^{3} I P}$.

Combining these equations, one finds the spin-down time scale $\left(\tau_{\mathrm{a}}=P / 2 \dot{P}\right)$ as

$\tau_{\mathrm{a}} \simeq 1.2 \times 10^{5} \mu_{30.3}^{-1} I_{45} \dot{M}_{17.5}^{-1 / 2} V_{7}^{-1 / 2} \mathrm{yr}$,

where $V_{7}=V_{\text {rel }} / 10^{7} \mathrm{~cm} \mathrm{~s}^{-1}$.

\subsection{Supersonic propeller spin-down time scale}

The spin-down rate of a star in the state of supersonic propeller is

$\dot{P}=\frac{P^{3} L_{\text {sups }}}{4 \pi^{2} I}$,

where $L_{\text {sups }}$ is the rate of the rotational energy loss by the star in the state of supersonic propeller. In the general case, this value can be limited to $\lesssim \dot{M} G M / R_{\mathrm{m}}$. Therefore, combining Eqs. (6) and (9), one can express the lower limit to the spin-down time scale of stars in the supersonic propeller state as

$\tau_{\mathrm{c}} \simeq 8 \times 10^{4} \mu_{30.3}^{-3 / 7} I_{45} \dot{M}_{17.5}^{-11 / 14} V_{7}^{1 / 2} \mathrm{yr}$.

Although our estimate represents the absolute minimum for $\tau_{\mathrm{c}}$, the real value of this parameter is unlikely to be significantly larger. In particular, as shown by Lipunov \& Popov (1995), the duration of the supersonic propeller state under all reasonable conditions turns out to be smaller than the spin-down time scale of the star in the state of ejector. On this basis, one can limit the age of the star as it has evolved to the state of subsonic propeller as

$\left(\tau_{\mathrm{a}}+\tau_{\mathrm{c}}\right) \lesssim \tau \lesssim 2 \tau_{\mathrm{a}}$.

\subsection{Subsonic propeller spin-down time scale}

Finally, the spin-down time scale of the star in the subsonic propeller state can be expressed following Ikhsanov (2001b, Eq. (10)), as

$\tau_{\mathrm{d}} \simeq 2 \times 10^{3} \mu_{30.3}^{-2} I_{45} P_{5} m_{1.4} \mathrm{yr}$,

where $m_{1.4}=1.4 M_{\odot}$.

$\tau_{\mathrm{d}}$ is small compared to the value of both, $\tau_{\mathrm{a}}$ and $\tau_{\mathrm{c}}$. Hence, the age of the neutron stars with the parameters derived in Sect. 2 is determined mainly by the pulsar-like spin-down time scale and can be limited to $<2 \tau_{\mathrm{a}}$. In order to fit the currently adopted evaluations of the ages of AXPs, one has to suggest that in a previous epoch the conditions in the circumstellar environment were slightly different. In particular, one could assume that the strength of the wind in the previous epoch was about $10^{18} \mathrm{~g} \mathrm{~s}^{-1}$, which corresponds to the accretion process at the Eddington limit. On the other hand, one can also envisage a situation in which the value of the relative velocity $V_{\text {rel }}$ is slightly larger than $100 \mathrm{~km} \mathrm{~s}^{-1}$ (this normalization of the velocity comes from the condition for the self-consistency of the supersonic propeller model, see Eq. (7) in Ikhsanov 2002). In this case the age of subsonic propellers turns out to be close to the typical ages of the SNRs with which they are presently associated. 


\section{Discussion}

As shown above, some basic features of AXPs can be explained in terms of neutron stars in the state of subsonic propeller provided their mass and magnetic moment are $M \sim 1.4 M_{\odot}$ and $\mu \sim 2 \times 10^{30} \mathrm{G} \mathrm{cm}^{3}$, and the strength of the wind is $\dot{M}_{\mathrm{c}} \sim 3 \times 10^{17} \mathrm{~g} \mathrm{~s}^{-1}$. The required values of mass and dipole magnetic moment are typical for neutron stars. The required strength of the wind, however, is rather high and this condition appears to be a strong restriction on the applicability of the proposed scenario. Indeed, for the wind strength $\sim 10^{17} \mathrm{~g} \mathrm{~s}^{-1}$ to realize, the neutron star should be embedded in a medium of density

$\rho \sim 10^{-16} \dot{M}_{17} M_{1.5}^{-2} V_{7}^{3} \mathrm{~g} \mathrm{~cm}^{-3}$.

Even in the case of a low relative velocity $V_{\text {rel }} \sim 10^{6} \mathrm{~cm} \mathrm{~s}^{-1}$ (which is about the sound speed at the accretion radius of the star), the required number density of the surrounding gas, $N_{\mathrm{e}} \sim$ $10^{5} \mathrm{~cm}^{-3}$, remains significantly larger than the average density of material in SNRs and ISM. Hence, for the proposed scenario to be applicable one has to assume that AXPs are embedded in relatively dense regions.

What are the physical conditions in these regions? As follows from X-ray observations, the column density in the observed AXPs is $(0.4-2) \times 10^{22} \mathrm{~cm}^{-2}$ (see e.g. Table 2 in Mereghetti et al. 2002). This indicates that the column density in the dense region is limited to

$N_{\mathrm{e}} L \lesssim 4 \times 10^{21} \mathrm{~cm}^{-2}$,

where $N_{\mathrm{e}}$ is the number density of the material in the dense region and $L$ is the size of the region along the line of sight.

If we require the life time of the accreting X-ray source to exceed the spin-down time scale of the subsonic propeller, one gets the following condition:

$N_{\mathrm{e}} m_{\mathrm{p}} \Phi \gtrsim \frac{L_{\mathrm{acc}} R_{\mathrm{ns}}}{G M_{\mathrm{ns}}} \tau_{\mathrm{d}}$

where $\Phi$ is the volume of the dense region.

Finally, the total flux emitted by the material accumulated in the dense region should not exceed the fluxes detected from the infrared counterparts of AXPs. Under the conditions of interest, the dense regions are optically thin at the frequencies $10^{14}-10^{15} \mathrm{~Hz}$. Hence, using the data presented in Fig. 2 of Israel et al. (2004) one finds:

$N_{\mathrm{e}}^{2} T^{1 / 2} \Phi \lesssim 6 \times 10^{58}$

where $T$ is the average temperature of the material in the dense region.

Combining Eq. (14)-(16) and setting $T \sim 2000 \mathrm{~K}$ (the error of a factor of 2 in this parameter does not affect the final conclusions) we find that the subsonic propeller approach to the interpretation of AXPs is applicable if the corresponding X-ray sources are embedded in regions of volume $\Phi \sim 5 \times 10^{42} \mathrm{~cm}^{3}$, and average number density $N_{\mathrm{e}} \sim 10^{7} \mathrm{~cm}^{-3}$. The size of the regions along the line of sight is limited to $L \lesssim 7 \times 10^{13} \mathrm{~cm}$. Under these conditions the appearance of subsonic propellers is in agreement with properties of AXPs in both the X-rays and the infrared part of the spectrum.
The question about the possible origin of such regions is rather complicated. In contrast to models built around the assumption that neutron stars in AXPs are in the state of accretor, the gravitational potential of the star within our scenario does not play a central role in the formation of its dense environment. Indeed, the required size of the environment significantly exceeds the accretion radius of the star. This indicates that the formation of the dense region is rather independent of the properties of the neutron star itself, but is related to the parameters of the supernova explosion or the properties of its progenitor or both.

Three possible scenarios for the formation of dense environments around AXPs has recently been outlined by Marsden et al. (2001): "fall-back" disk accretion, "pushed-back" disk accretion, and accretion involving high-velocity neutron stars. The fall-back disk accretion scenario within the subsonic propeller approach can be rejected since the material stored in the disk is assumed to be gravitationally bound to the star (this disk is situated within the accretion radius of the star, see e.g. Chatterjee et al. 2000, and references therein).

The scenario in which a high-velocity neutron star captures material from the co-moving ejecta can be rejected on the same basis. Indeed, the number density in the expanding ejecta is $n \propto t^{-3}$ and therefore the life-times of AXPs within the subsonic propeller scenario turn out to be too short. The life-time could be longer if the material captured by the star were stored in a disk within its gravitational radius, but this assumption contradicts the basic statements of our model.

Within the pushed-back disk scenario the dense environment of the star is expected to form on a time scale of $10^{3} \mathrm{yr}$. The total amount of material stored in such a disk is about $0.4 M_{\odot}$ (see Marsden et al. 2001, and references therein) and the disk is not gravitationally bound to the neutron star. Unfortunately, the basic properties of such a disk have been investigated rather poorly. For this reason, a detailed analysis of the applicability of this model to the subsonic propeller approach is presently not possible. Nevertheless, the above mentioned estimates of the mass and the time scale indicate that the pushed-back disk scenario could be a solution for the problem of the origin of the dense environment required in our model.

It should also be noted that both the identification of the infrared counterparts of AXPs (Israel et al. 2004) and the conclusion about the compact geometry of their host SNRs (Marsden et al. 2001) give some hints that there exists a dense environment surrounding these objects. In the light of this, the requirements of our model do not seem to be artificial but turn out to be rather reasonable from the observational point of view.

\section{Conclusion}

The appearance of magnetized $\left(B \sim 3 \times 10^{12} \mathrm{G}\right)$ neutron stars in the state of subsonic propeller, which are situated in a strong $\left(\dot{M}_{\mathrm{c}} \sim 3 \times 10^{17} \mathrm{~g} \mathrm{~s}^{-1}\right)$ wind, is similar to that of AXPs in several important aspects. Namely, the rotational periods of these objects are limited to the range of 5-15 s, and the spin-down rates are about $7 \times 10^{-11} \mathrm{~s} \mathrm{~s}^{-1}$. The emission of stars in this state is partly $(5-10 \%)$ contributed to by the hot envelope surrounding its magnetosphere and mainly (90-95\%) by the process of 
accretion of material onto the stellar surface. The efficiency of the accretion process, which is governed by the reconnection of the magnetic field lines at the magnetospheric boundary, is high enough for the luminosity of the considered objects to be of order a few $\times 10^{35} \mathrm{erg} \mathrm{s}^{-1}$. Finally, the estimated ages of neutron stars under the conditions of interest are close to $10^{5} \mathrm{yr}$.

The above derived parameters allow us to suggest that the interpretation of AXPs within the subsonic propeller approach is rather promising for the future investigations.

Acknowledgements. We would like to thank the anonymous referee for useful comments. The work was partly supported by the Russian Foundation of Basic Research under grant 03-02-17223a, and the State Scientific and Technical Program "Astronomy".

\section{References}

Arons, J., \& Lea, S. M. 1976a, ApJ, 207, 914

Arons, J., \& Lea, S. M. 1976b, ApJ, 210, 792

Davies, R. E., \& Pringle, J. E. 1981, MNRAS, 196, 209
Chatterjee, P., Hernquist, L., \& Narayan, R. 2000, ApJ, 534, 373

Colpi, M., Geppert, U., \& Page, D. 2000, ApJ, 529, L29

Eksi, K. Y., \& Alpar, M. A. 2003, ApJ, 599, 450

Elsner, R. F., \& Lamb, F. K. 1976, Nature, 262, 356

Ikhsanov, N. R. 2001a, A\&A, 375, 944

Ikhsanov, N. R. 2001b, A\&A, 368, L5

Ikhsanov, N. R. 2002, A\&A, 381, L61

Ikhsanov, N. R. 2003, A\&A, 399, 1147

Israel, G. L., Stella, L., Covino, S., et al. 2004, in Young Neutron Stars and Their Environments, ed. F. Camilo, \& B. M. Gaensler, IAU Symp., 218, in press [arXiv: astro-ph/0310482]

Lipunov, V. M., \& Popov, S. B. 1995, Astron. Rep., 39, 632

Marsden, D., Lingenfelter, R. E., Rotschild, R. E., \& Higdon, J. C. 2001, ApJ, 550, 397

Mereghetti, S., Chiarlone, L., Israel, G. L., \& Stella, L. 2002, in Neutron Stars, Pulsars and Supernova Remnants, ed. W. Becker, H. Lesch, \& J. Trum̈per, MPE Rep., 278, 29

Priest, E. R., \& Forbes, T. G. 2000, Magnetic reconnection: MHD theory and applications (Cambridge University Press)

Psaltis, D., \& Miller, M. C. 2002, ApJ, 578, 325

Thompson, C., \& Duncan, R. C. 1996, ApJ, 473, 322

Wang, Y.-M., \& Robertson, J. A. 1985, A\&A, 151, 361 